\title{
Qualidade de energia: estudo de caso de uma indústria metalmecânica no sul do Brasil
}

\author{
Bruna Steil Boneberg \\ Matheus Berghan \\ Lucas de Matos \\ Marcelo Leal Dellinghausen \\ Vinicius Leônidas Curcio \\ Elton Gimenez Rossini
}

Universidade Estadual do Rio Grande do Sul (UERGS), Novo Hamburgo, RS, Brasil E-mails: bruna.steil.boneberg@gmail.com matheusberghan@gmail.com lucasdmatos@gmail.com mdellinghausen@hotmail.com vinicius-curcio@uergs.edu.br elton-rossini@uergs.edu.br

Recebido em: 27 mar. 2017. Aceito: 22 maio 2017.

DOI: http://dx.doi.org/10.21674/2448-0479.32.315-339

\section{Resumo}

Atualmente, com o aumento do consumo de energia no mundo, a sociedade vem a cada dia se preocupando mais com as medidas de uso racional de energia. O fornecimento de energia é um dos serviços mais desafiantes da sociedade moderna, onde falhas momentâneas podem provocar elevados prejuízos. Há uma grande preocupação com a qualidade no fornecimento e na forma de utilização da energia elétrica no setor industrial, o qual possui demasiada importância, uma vez que a energia elétrica consumida no setor industrial brasileiro corresponde a cerca de $46 \%$ da demanda total do país. A análise da qualidade de energia no setor industrial, apresenta-se como uma oportunidade de gestão energética que pode gerar inúmeros benefícios, como por exemplo servir como um instrumento de competitividade no mercado, pois evita o desperdício de energia elétrica e favorece a vida útil de maquinários. Este artigo visa a anaRev. Elet. Cient. UERGS, v. 3, n. 2, p. 315-339, 2017 
lisar a qualidade da energia elétrica, primeira etapa de uma auditoria energética, utilizada por uma empresa situada na região do Vale dos Sinos no Estado do Rio Grande do Sul. O estudo de caso aborda fatores relacionados à qualidade da energia elétrica da empresa, tais como tensão de regime permanente, flutuação de tensão, corrente e fator de potência, que foram obtidos a partir da análise de 8644 registros, totalizando 144 horas de medições.

Palavras-chave: Auditoria Energética. Diagnóstico Energético. Análise Industrial. Qualidade de Energia. Banco de Capacitores.

\section{Abstract}

\section{Power Quality: a case study of a machining company of the industrial sector in the South of Brazil}

Nowadays, with the growth in energy consumption in the world, the society has increasingly concerned with rational energy use measures. Energy supply is one of the most challenging services in modern society, where momentary failures can cause heavy losses. There is a great concern about the quality of supply and the use of electricity in the industrial sector, which gains importance, since the electric energy consumed in the Brazilian industrial sector corresponds to about $46 \%$ of the total demand of the country. The analysis of energy quality in the industrial sector presents itself as an energy management opportunity that can generate numerous benefits, such as serving as an instrument of competitiveness in the market, as it avoids the waste of electricity and prolong life of machinery. This paper study intends to analyse the quality of electric energy, the 
first stage of an energy audit, used by a company located at Vale dos Sinos region in the State of Rio Grande do Sul, Brazil. The case study addresses factors from 8644 records, totalizing 144 hours of measurements, related to energy quality, such as voltage, current and power factor.

Keywords: Energy Audit. Energy diagnostic. Industrial Analysis. Power Quality. Capacitors bank.

\section{Introdução}

A energia é um insumo essencial e o seu uso final na classe industrial representa $46 \%$ do total consumido, sendo também responsável por expressiva parcela de desperdício (BELINOVSKI; BISPO; SILVA, 2009; ELETROBRÁS, 2006). Segundo Mamede Filho (2015) os motores elétricos numa instalação industrial consomem em média de $75 \%$ da energia de demanda. A auditoria energética surge como uma ferramenta efetiva para contabilizar o consumo de energia, a eficiência energética dos seus equipamentos e as perdas implicadas, que através de uma análise aprofundada da qualidade de energia, tem como finalidade amortizar os custos sem prejudicar a produção (LOURENÇO, 2009). Além disso, como etapa inicial de uma auditoria, a qualidade da energia elétrica garante a performance e vida útil de equipamentos, tornando-se vital para a indústria metalmecânica que depende diretamente da linha de produção de equipamentos de grande porte, normalmente.

Conforme Nascimento (2014), uma auditoria energética consiste em um levantamento aprofundado da situação energética atra- 
vés da análise das quantidades de energia utilizadas em cada uma das operações do processo de fabrico. De acordo com Gaspar (2004), o objetivo de uma auditoria é apoiar o gestor de energia na seleção tecnológica mais adequada para possíveis investimentos em uma utilização racional da energia. Ainda, os dados recolhidos numa auditoria energética permitem estabelecer um conjunto de medidas conducentes à redução dos consumos energéticos da empresa. Entretanto, para atingir as metas estipuladas em uma auditoria, deve-se compreender o conceito e os parâmetros de qualidade de energia elétrica.

A análise da qualidade da energia elétrica aparece, por conseguinte, a partir do momento em que os consumidores constatam interrupções no fornecimento de energia sob o ponto de vista tecnológico (MEHL, 2012). A continuidade e conformidade da energia elétrica são fatores que influenciaram extremamente na qualidade de energia. Há diversos parâmetros que podem ser considerados na análise como, por exemplo, flutuação de tensão, variações de tensão de curta duração, desequilíbrio de sistemas trifásicos, entre outros (AGÊNCIA NACIONAL DE ENERGIA ELÉTRICA, 2016).

De acordo com Lima (2009), pode-se classificar como um distúrbio de qualidade de energia elétrica qualquer anomalia referente a tensão, corrente ou desvio de frequência, a qual acarrete problemas na operação de equipamentos elétricos. Deste modo, questões econômicas estão diretamente relacionadas com a qualidade da energia elétrica, uma vez que distúrbios corriqueiros poderão ocasionar mau funcionamento de equipamentos, aumentando o seu consumo, assim como causando possíveis avarias. Segundo Cruz (2007), a qualidade de energia elétrica que não atende aos requisitos básicos, estipulados pelo PRODIST - Modulo 8, pode gerar perdas tanto na produção, devido à ociosidade do processo, quanto nos 
equipamentos instalados em funcionamento, devido à falta de fornecimento de energia elétrica.

Devido à cobrança de valores adicionais pelas concessionárias de energia para os excedentes de demanda e consumo reativos, há uma constante preocupação de profissionais responsáveis pela manutenção, operação e gerenciamento de instalações industriais com relação à correção do fator de potência para atender o limite de referência estabelecido pela ANEEL - Agência Nacional de Energia Elétrica, em sua Resolução n456/2000 (CREDER, 2013).

Segundo Comitê de Distribuição de Energia Elétrica (2004), ao passo que a potência ativa é continuamente consumida na execução de trabalho, a potência reativa não produz trabalho e ocupa um "espaço" no sistema elétrico que poderia ser utilizado para o fornecimento de mais energia ativa, pois essa circula entre a carga e a fonte de alimentação. Ainda, o fator de potência (FP) indica o uso eficiente de energia elétrica, ou seja, FP é a relação entre a potência ativa e potência reativa, em outras palavras, é o cosseno do ângulo de defasagem entre a corrente e a tensão.

De acordo com Comitê de Distribuição de Energia Elétrica (2004) e Creder (2013), as causas mais comuns da ocorrência de baixo fator de potência são: motores e transformadores operando em vazio, com problemas de cargas, ou superdimensionados; grande quantidade de motores de pequena potência; lâmpadas de descarga (fluorescentes, vapor de mercúrio, vapor de sódio); e excesso de energia reativa.

A indústria metalmecânica abrange todos os segmentos responsáveis pela produção e transformação de metais que, por sua vez, compreende os setores de usinagem, estamparia, forjaria, montagem, etc. A diversidade dos produtos depende de processos e, principalmente, equipamentos de grande porte utilizados em uma 
linha de produção. Conforme Mamede Filho (2015), determinados equipamentos, tais como motores elétricos, necessitam para sua operação de uma certa quantidade de energia reativa, podendo ser indutiva ou capacitiva. Para isso, utiliza-se banco de capacitores em paralelo com a rede elétrica, devido ao baixo custo de instalação e manutenção.

O presente trabalho visa a analisar a qualidade da energia elétrica utilizada por uma empresa situada na região do Vale do Sinos, no Estado do Rio Grande do Sul, Brasil. O objetivo é analisar se os critérios designados pelo PRODIST, relacionados à qualidade de energia da empresa, estão sendo atendidos. Entre os dados analisados, temos classificação da tensão de atendimento, desequilíbrio de tensão, análise das correntes e o fator de potência.

\section{Materiais e Métodos}

A metodologia do trabalho elaborado na empresa baseou-se nos procedimentos orientados pelos documentos elaborados pela ANEEL - Procedimentos de Distribuição de Energia Elétrica no Sistema Elétrico Nacional - PRODIST.

Para a realização deste trabalho utilizou-se um registrador de energia modelo RMS MARH-21. Este analisador possui diversos modos de operação. O modo selecionado foi Medição e Registro de Grandezas Integralizadas. O período de integração dos registros foi entre o dia 7 de outubro de 2016, a partir das 10 horas da manhã, até o dia 13 de outubro de 2016 até o mesmo horário, ou seja, durante um período de 144 horas, com medições registradas a cada 60 segundos, totalizando 8644 medidas. 
As grandezas registradas pelo MARH-21 foram: tensões de fase (fase-neutro), fases a, b e c; correntes, fases a, b e c; potências ativas, fases a, b e c; potências reativas, fases a, b e c; distorção harmônica total de tensão, fases a, b e c; distorção harmônica total de corrente, fases a, b e c; máximo e mínimo de tensão ( $r m s, 1 / 2$ ciclo, com indicação da fase); máximo de corrente ( $\mathrm{rms}, 1 / 2$ ciclo, com indicação da fase); tempo com tensão fora da faixa programada; tempo com corrente acima do valor programado, frequência (fase a ou b ou c), grau de desequilíbrio de tensão. No entanto, apenas os dados de tensão de fase, desequilíbrio entre as tensões de linha, corrente de fase, e fator de potência foram estudados.

Sempre que ocorria o desligamento da chave geral no quadro de distribuição, o analisador sustentava a forma de registro e preenchimento de dados na memória, das $18 \mathrm{~h}$ às $7 \mathrm{~h}$ do dia seguinte, quando era acionada novamente. A taxa de amostragem do MARH21 é de 64 amostras/ciclo e apresenta uma precisão de leitura de $0,5 \%$. Na Figura 1 é apresentado o esquema de ligação no quadro geral da empresa, onde os símbolos $V_{A}, V_{B}$ e $V_{c}$ representam as garras de tensão do cabo de sinal do analisador, e os símbolos $I_{c}, I_{B} \mathrm{e}$ $I_{A}$ representam os alicates de corrente com as respectivas setas indicadoras do fluxo de energia do sistema.

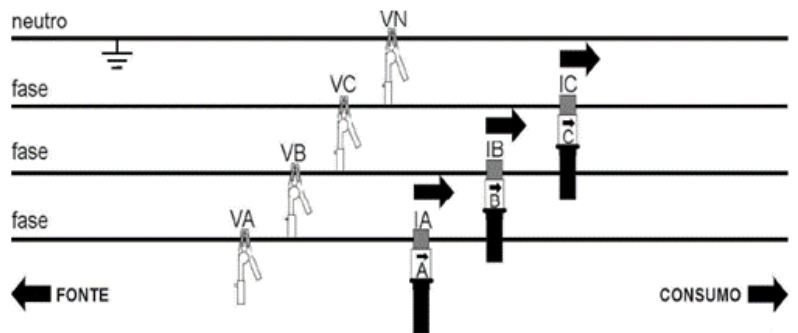

Figura 1 - Esquema de ligação aos sistemas de potência. Fonte: Adaptado de GSI Engenharia e Consultoria, [2017]. 
A respeito da instalação do equipamento no local, alguns cuidados foram adotados, como por exemplo: ao conectar as garras de tensão aos barramentos, iniciou-se com a garra de Neutro. Somente após a conexão da garra de Neutro é que as demais garras do cabo de sinal de tensão foram conectadas, começando pela garra $A$, depois a $B$ e então a $C$, independente da identificação de fases existente (ou não) no barramento. Foi essencial seguir as orientações mencionadas, já que facilitaria a identificação das fases durante 0 trabalho de análise de relatórios e gráficos via ANAWIN, o software disponibilizado pelo equipamento.

O software ANAWIN 4.14 (2013) foi fornecido juntamente com o registrador e possibilitou a análise dos dados de forma prática e direta através de gráficos e relatórios. Foi possível realizar a simulação com acréscimo de banco de capacitores, o que possibilitou verificar a quantidade de capacitores e, portanto, a energia reativa necessária para corrigir o fator de potência. Além do mais, alguns dados foram exportados para serem analisados no MS Excel e Libre Office Calc para gerar arquivos vetoriais.

\section{Informações da Empresa}

A empresa do estudo de caso, cujo nome é mantido em sigilo por questões de confidencialidade, é localizada na região do Vale dos Sinos no Estado do Rio Grande do Sul. A empresa conta com $4.000 \mathrm{~m}^{2}$ de área construída, com um parque de máquinas composto por setor de ferramentaria, que inclui ferramentaria produtiva, desenvolvimento e manutenção de dispositivos (matrizes para conformação de barras e tubos), célula de máquinas operatrizes, cen- 
tros de usinagem, tornos CNC e um laboratório completo de metrologia.

Entre os serviços prestados pela empresa podemos destacar trabalhos desenvolvidos como usinagem de precisão, usinagem para manutenção, produção de conjuntos montados e soldados, desenvolvimento de peças fundidas, forjadas e micro fundidas, além de peças com acabamento superficial e tratamento térmico.

\section{Dados de Consumo}

Conforme a proposta do trabalho, os dados analisados foram medidos entre os dias 7 a 13 de outubro de 2016. Após a segunda visita na empresa foi possível coletar algumas informações relevantes. Por exemplo, a fatura de energia disponibilizada pela concessionária AES SUL Distribuidora Gaúcha de Energia S.A.

O histórico de consumo e demanda entre outubro de 2015 e outubro de 2016, pode ser visualizado na Figura 2. Ainda, para um melhor entendimento da instalação, os dados essências da fatura de energia sāo:

a) Transformador: $23000 \mathrm{~V} / 380 \mathrm{~V}-220 \mathrm{~V}$.

b) Tensão nominal contratada: $23 \mathrm{kV}$.

c) Limites adequados: $21.39 \mathrm{KV}$ a $24.15 \mathrm{kV}$.

d) Tipo de uso: industrial.

e) Tarifa: VCM-HV Dem. Classes (13.8 a 23kV).

f) Demanda contratada fora de ponta: $80 \mathrm{kV}$.

g) Consumo médio fora de ponta: $13.290 \mathrm{kWh} / \mathrm{mês.}$

h) Consumo médio horário de ponta: 487,15 kWh/mês.

i) Consumo fora de ponta: $172.758 \mathrm{kWh} / \mathrm{ano}$. 
j) Consumo horário de ponta: 5845,8 kWh/ano.

k) Custo com Fatura de Energia Reativa (FER): +/- $\mathrm{R} \$ 400,00$ mês.

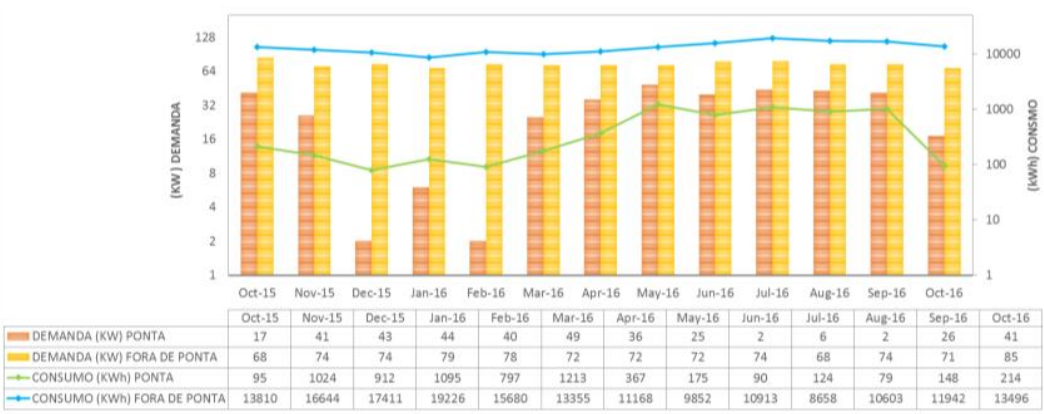

Figura 2 - Histograma do Consumo de Energia.

\section{Análise de Resultados}

Para a análise da qualidade de energia durante o período determinado, foram realizadas 8.644 medições, totalizando 144 horas.

\section{Tensão Nominal e Desequilíbrio de Tensão}

De acordo com Agência Nacional de Energia Elétrica (2016), o parâmetro usado para avaliar a qualidade da tensão contratada, nos pontos de conexão pelos usuários atendidos em tensão nominal de operação superior a $1 \mathrm{kV}$, deve se situar entre 95\% e $105 \%$ da tensão nominal de operação do sistema no ponto de conexão e, ainda, coincidir com a tensão nominal de um dos terminais de deri- 
vação previamente exigido ou recomendado para o transformador da unidade consumidora (AGÊNCIA NACIONAL DE ENERGIA ELÉTRICA, 2016). Ainda, segundo a ANEEL Resolução 505 Artigo $4^{\circ}$ de 26 de novembro de 2001, os limites máximos e mínimos, respectivamente, são $231 \mathrm{~V}$ e $201 \mathrm{~V}$.

Os valores estudados pelo software ANAWIN são exibidos na Figura 3, que apresentam os valores máximos e mínimos atingidos pelas tensões por fase, assim como as médias durante o período medido. Os valores máximos considerados no estudo são registrados apenas quando não há demanda por carga.

\begin{tabular}{|c|c|c|c|c|c|c|}
\hline \multicolumn{7}{|c|}{ Tensões } \\
\hline \multirow{2}{*}{$\begin{array}{l}\text { ENTRAOA } \\
\text { Muda (V) }\end{array}$} & \multicolumn{3}{|c|}{ Minima(S) } & \multicolumn{3}{|c|}{ MAXoma(S) } \\
\hline & HORA & DATA & $\mathrm{v}$ & HORA & DATA & $\mathrm{v}$ \\
\hline A: 231.88 & $\begin{array}{r}08: 07: 00 \\
11: 04: 00 \\
11: 27: 00\end{array}$ & $\begin{array}{l}12 / 1016 \\
11 / 1016 \\
11 / 1016\end{array}$ & $\begin{array}{l}214.81 \\
221.28 \\
221.35\end{array}$ & $\begin{array}{l}22 \cdot 56: 00 \\
02.57: 00 \\
22.54: 00\end{array}$ & $\begin{array}{l}08 / 10 / 16 \\
12 / 10 / 16 \\
08 / 10 / 16\end{array}$ & $\begin{array}{l}238.55 \\
238.38 \\
238.19\end{array}$ \\
\hline B: 23078 & $\begin{array}{r}06.07: 00 \\
11: 04: 00 \\
11.27: 00\end{array}$ & $\begin{array}{l}\text { 121016 } \\
11 / 1016 \\
11 / 1016\end{array}$ & $\begin{array}{l}215.49 \\
220.88 \\
220.96\end{array}$ & $\begin{array}{l}02.57: 00 \\
02.53: 00 \\
02.56 .00\end{array}$ & $\begin{array}{l}12 / 10 / 16 \\
12 / 10 / 16 \\
12 / 10 / 16\end{array}$ & $\begin{array}{l}237.11 \\
237.02 \\
236.87\end{array}$ \\
\hline C: 232.67 & $\begin{array}{r}08.07: 00 \\
11: 04: 00 \\
11: 27: 00\end{array}$ & $\begin{array}{l}12 / 1016 \\
11 / 1016 \\
11 / 1016\end{array}$ & $\begin{array}{l}216.36 \\
222.61 \\
222.66\end{array}$ & $\begin{array}{l}02.57 .00 \\
02.53 .00 \\
22.56 .00\end{array}$ & $\begin{array}{l}12 / 10 / 16 \\
12 / 10 / 16 \\
0810 / 16\end{array}$ & $\begin{array}{l}239.03 \\
238.94 \\
238.77\end{array}$ \\
\hline
\end{tabular}

Figura 3 - Relatório dos dados de Tensão do software RMS ANAWIN.

Através da verificação dos dados obtidos com relação aos valores nominais das tensões de fase, pode-se concluir que os valores permanecem dentro dos limites estabelecidos pela ANEEL, durante o horário de funcionamento da empresa, ou seja, das $7 \mathrm{~h} 30 \mathrm{~min}$ às 17h00min considerado fora de ponta, conforme Figura.4. Quando não há expediente na empresa, por consequência, as máquinas estão desligadas, o valor máximo de $231 \mathrm{~V}$ é ultrapassado, ou seja, há sobretensão, conforme apresentado na Figura 4. (b, c), também pelos dados de Sábado e Domingo, cujo não há expediente que difere do comportamento dos dias da semana na Figura 4. (a, d, e, f, 
g). Já na Figura 4. (h), é possível visualizar a variação das tensões de fase em percentuais e momentos em que os limites são ultrapassados.

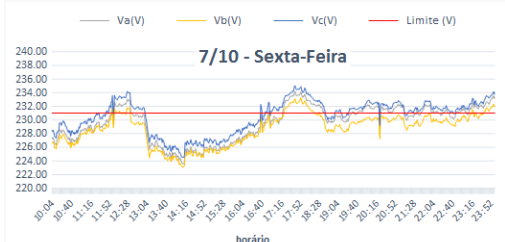

(a)

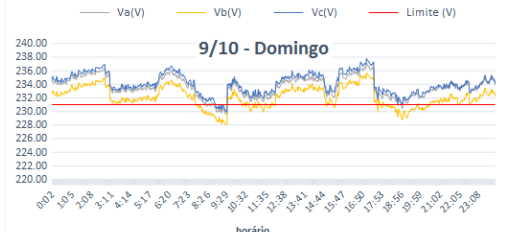

(c)

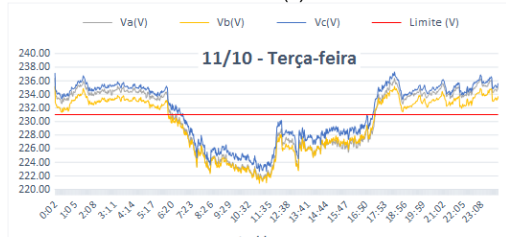

(e)

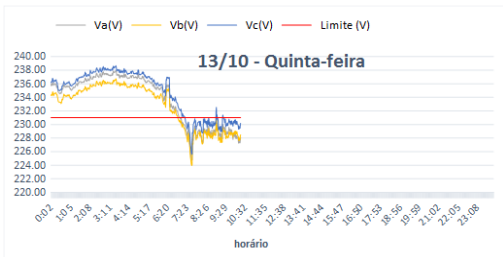

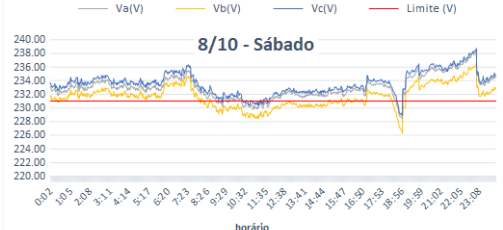

(b)

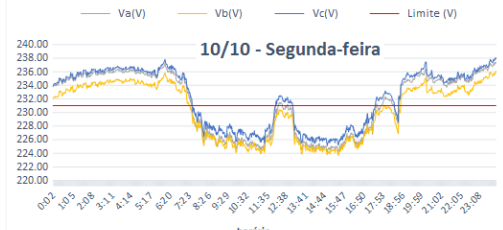

(d)

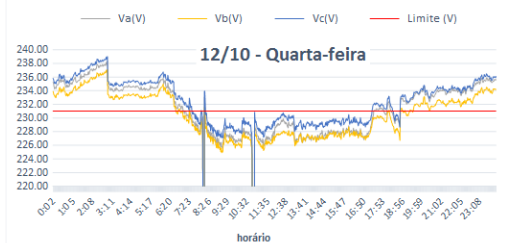

(f)

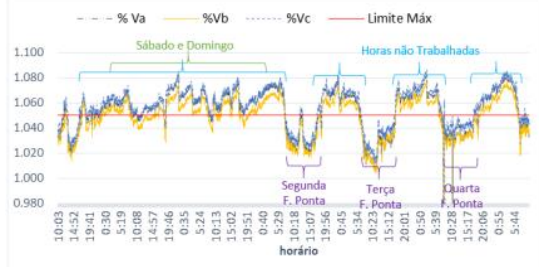

Figura 4 - (a)(b)(c)(d)(e)(f)(g) Variaçōes das tensões $V a, V b$ e $V c$ ao longo do dia. (h) Oscilações dos percentuais das tensões \% $\mathrm{Va}, \% \mathrm{Vb}$ e \% $\mathrm{Vc}$ ao longo de 6 dias ultrapassando o limite máximo de $105 \%$.

Conforme Operador Nacional do Sistema Elétrico (2016b), o desequilíbrio de tensão (D) entre fases pode ser determinado atra- 
vés do limite global nos barramentos da rede básica e nos barramentos dos transformadores de fronteira, devendo ser menor ou igual a 2\%, através da Equação 1.

$$
D(\%)=(\text { Vmaior } / \text { Vmenor })-1 \times 100 \%
$$

Para calcular os desequilíbrios de tensão de linha, deve-se determinar a maior tensão - $V_{\text {maior }}$ - assim como a menor tensão $V_{\text {menor }}$ - para um determinado período, onde há três valores de tensão $V_{A}, V_{B}$ e $V_{C}$.

Os valores calculados referentes aos desequilíbrios de tensão variam em torno de 5,4\%. Este problema é agravado quando consumidores com alimentação trifásica apresentam uma distribuição de carga ineficiente, impondo correntes desequilibradas no circuito da concessionária.

Outra justificativa para o problema, o transformador da empresa pode estar realizando a conversão para uma tensão maior no secundário do transformador. Ou seja, o TAP do transformador, que permite elevar ou reduzir a tensão no secundário, através da variação da relação de transformação. Neste caso, a empresa deverá entrar em contato com uma empresa especializada e autorizada pela concessionária para realizar a verificação deste desequilíbrio.

Na prática, a empresa deve solicitar o desligamento da linha de alimentação de média tensão para a concessionária. Em seguida, de ser executado os procedimentos de segurança conforme Brasil (2004), a NR 10 - Segurança em Instalações e Serviços em Eletricidade: constatação da ausência de tensão, instalação do aterramento temporário, entre outras medidas de segurança.

Para verificação a relação de transformação, efetua-se um ensaio elétrico com um equipamento conhecido como TTR. Este equi- 
pamento injeta uma tensão no primário e faz a leitura da tensão do secundário. Depois, com os valores obtidos é calculado a relação de transformação e comparado o valor com a relação de transformação do transformador.

\section{Análise das Correntes la, lb e Ic}

Com relação à análise de correntes, o desequilíbrio máximo de corrente entre fases aceito deve ser até $10 \%$, valor definido por normas internacionais IEEE Standard 519 (1992) e IEC 61000-3-4 (EMC) de 1998. Conforme mostrado na Figura 5 (a,b,c,d,e,f,g), é possível acompanhar o comportamento das correntes de fase $l_{a}, l_{b}, l_{c}$ e, ainda, confirmar que o horário de intervalo ocorre entre $12 \mathrm{~h}$ e $13 \mathrm{~h}$ durante os dias da semana. No dia 12 de outubro de 2016 ocorreram duas interrupções no fornecimento de energia entre 8h08min e $8 \mathrm{~h} 11 \mathrm{~min}$, assim como entre $10 \mathrm{~h} 48 \mathrm{~min}$ e $10 \mathrm{~h} 54 \mathrm{~min}$, no entanto foram diagnosticadas como responsabilidade da concessionária, nada fora do limite permitido pelo contrato de atendimento.

Os dados obtidos pelo software ANAWIN são exibidos na Figura 6. São apresentados os valores máximos e mínimos atingidos pelas correntes por fase, assim como as médias durante o período medido. A partir dos dados medidos, pode-se perceber que a princípio na empresa, não há uma programação para acionar os equipamentos em diferentes horários, portanto, quando vários motores e outros equipamentos iniciaram a operação de forma simultânea, ocorreu o retorno da energia comercial ocasionando os picos de corrente registrados.

Ainda, os valores máximos de corrente registrados ocorreram durante um período menor que 8 segundos, de maneira que não 
desarmou o disjuntor, cuja curva de ruptura pode ser visualizada na Figura 7.

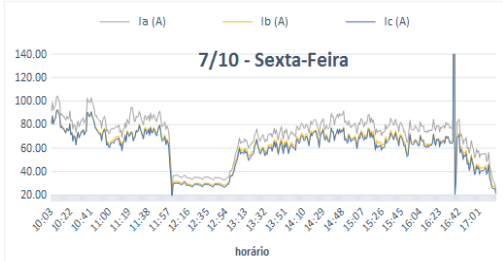

(a)

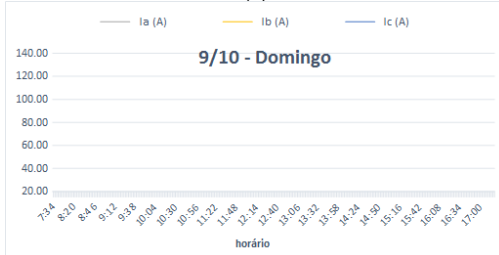

(c)

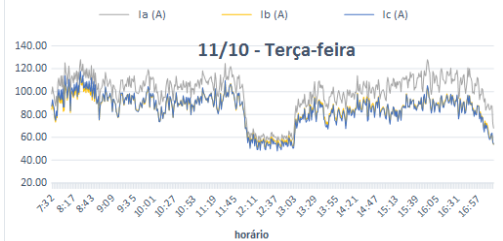

(e)

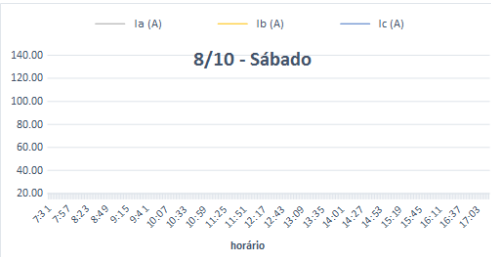

(b)

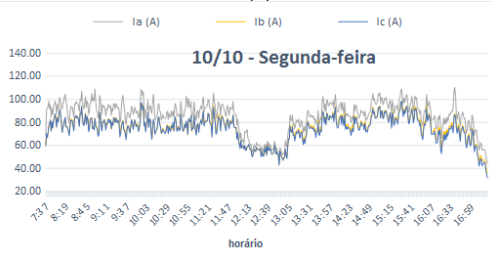

(d)

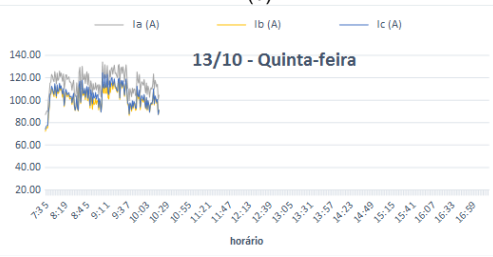

(f)

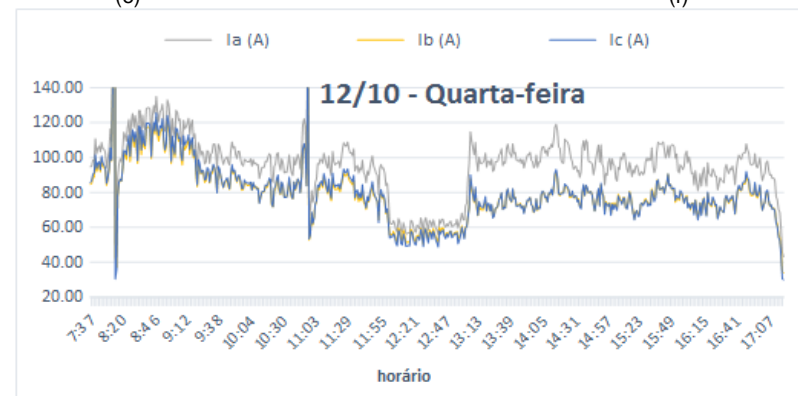

Figura 5 - $(a)(b)(c)(d)(e)(f)(g)$ Variaçōes das correntes la, lb e Ic. 


\begin{tabular}{|c|c|c|c|c|c|c|}
\hline \multicolumn{7}{|c|}{ Correntes } \\
\hline \multirow{2}{*}{$\begin{array}{l}\text { ENTRADA } \\
\text { Midia (A) }\end{array}$} & \multicolumn{3}{|c|}{ MiNIMA(\$) } & \multicolumn{3}{|c|}{ Máxime(\$) } \\
\hline & HORA & DATA & A & HOPA & DATA & A \\
\hline A: 31.785 & $\begin{array}{l}18: 20.00 \\
18: 19.00 \\
18: 18.00\end{array}$ & $\begin{array}{l}\text { 11/1016 } \\
11 / 1016 \\
11 / 1016\end{array}$ & $\begin{array}{l}3.000 \\
3.000 \\
3.000\end{array}$ & 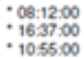 & $\begin{array}{l}1210 / 16 \\
071016 \\
12 / 10 / 6\end{array}$ & $\begin{array}{l}835.25 \\
329.80 \\
187.92\end{array}$ \\
\hline B: $27: 203$ & $\begin{array}{l}08: 56: 00 \\
18.32: 00 \\
18: 28.00\end{array}$ & $\begin{array}{l}121016 \\
11 / 1016 \\
11 / 1016\end{array}$ & $\begin{array}{l}2.644 \\
2644 \\
2644\end{array}$ & $\begin{array}{r}08: 12.00 \\
: 1637.00 \\
\cdot 1055: 00\end{array}$ & $\begin{array}{l}1210 / 16 \\
07 / 10 / 16 \\
12 / 10 / 16\end{array}$ & $\begin{array}{l}876.59 \\
400.81 \\
144.46\end{array}$ \\
\hline C: 27.328 & $\begin{array}{l}17: 36: 00 \\
17: 33: 00 \\
17: 32: 00\end{array}$ & $\begin{array}{l}07 / 1016 \\
0711016 \\
07 / 1016\end{array}$ & $\begin{array}{l}2.975 \\
2.975 \\
2975\end{array}$ & 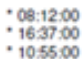 & $\begin{array}{l}1210 / 16 \\
0710016 \\
121016\end{array}$ & $\begin{array}{l}503.64 \\
337.19 \\
15928\end{array}$ \\
\hline
\end{tabular}

Figura 6 - Relatório dos dados de Correntes do software RMS ANAWIN.

Já na Figura 7, temos a curva do modelo dos disjuntores do quadro geral da empresa, disjuntor termomagnético Soprano 200A Nema AB1. De acordo com a curva do mesmo, podemos concluir que se o valor da corrente fosse cinco vezes maior que 200A, o tempo de operação limite mínimo do disjuntor para desligar o fornecimento de corrente seria de 10 segundos. Logo, as oscilações extremas, mencionadas na Figura 6, respeitaram os limites de segurança do disjuntor.

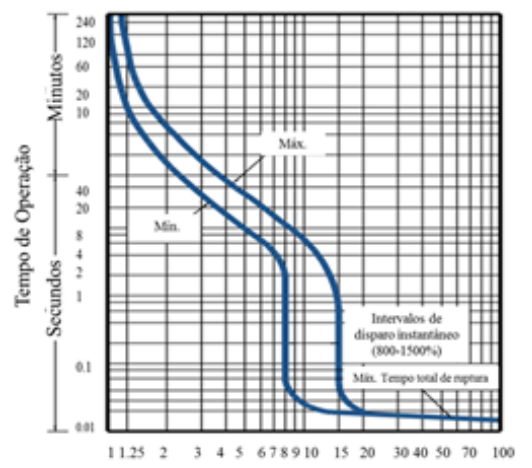

$\mathrm{Ix}$

Figura 7- Curva do Disjuntor Soprano padrão NEMA.

Fonte: Adaptado de Soprano, [2011]. 
Na Figura 8 é apresentado um histograma de todo o período medido. Pode-se observar que há uma sobrecarga em uma das fases durante o horário de trabalho - vide a variável $I_{x}$.

A solução mais econômica seria uma readequação dos circuitos da empresa para promover uma distribuição mais uniforme de cargas, de modo a reduzir o desequilíbrio.

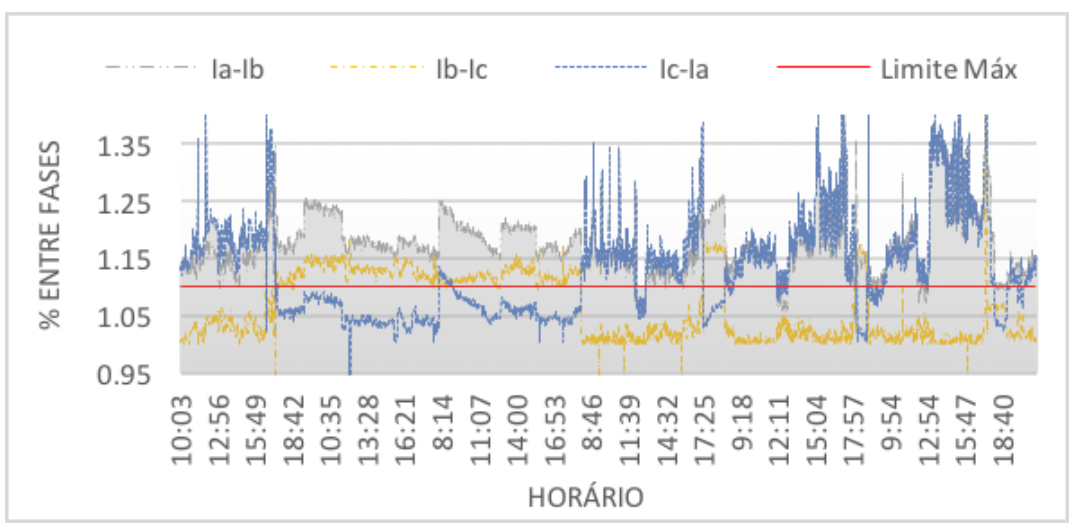

Figura 8 - Histograma do Consumo de Energia em percentual (\%).

\section{Fator de Potência}

O valor do fator de potência deverá ser calculado a partir dos valores registrados das potências ativa e reativa $(P, Q)$ ou das respectivas energias (EA, ER) energia ativa e reativa. Neste caso, utilizando-se a fórmula:

$$
f p=P / \sqrt{P^{2}+Q^{2}}
$$

É fundamental, para unidade consumidora ou conexão entre distribuidoras com tensão inferior a $230 \mathrm{kV}$, que o fator de potência 
no ponto de conexão esteja compreendido entre 0,92 e 1,00 indutivo ou 1,00 e 0,92 capacitivo, de acordo com regulamentação vigente (AGÊNCIA NACIONAL DE ENERGIA ELÉTRICA, 2016).

$\mathrm{Na}$ Figura 9 são mostrados os valores obtidos pelo software ANAWIN para os fatores de potência da empresa durante o período das medições. Claramente não atende os critérios estabelecidos, todos os valores estão abaixo de 0,92 . Por isso, a empresa pagou multas durante o ano de 2016 devido aos excedentes de energia reativa indutiva, totalizando um custo adicional médio de $\mathrm{R} \$ 5.000,00$ por ano.

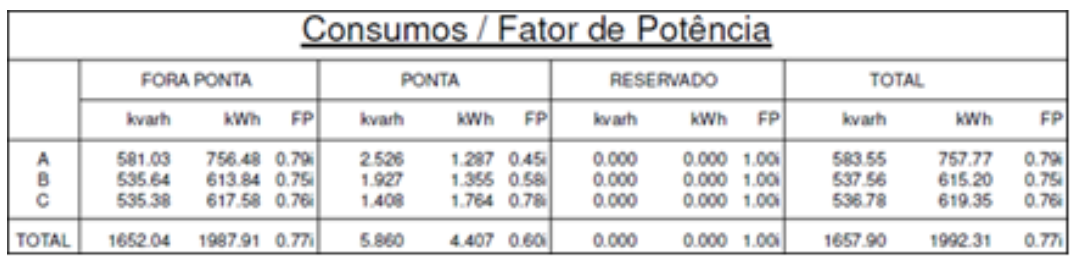

Figura 9 - Relatório dos dados de Fator de Potência do software RMS ANAWIN.

O comportamento do fator de potência das fases ilustrado na Figura 10, permite acompanhar as oscilações durante o período de expediente dos dias úteis. Sem exceção, os registros não atenderam as condições de operação estabelecidas pela ANEEL.

Já na Figura 11, pode-se visualizar que através de simulações no software RMS ANAWIN, foi possivel obter valores para os fatores de potência equivalente a 1 acrescentando ao projeto um banco de capacitores trifásicos de 10kVAr por fase. No entanto, para evitar valores capacitivos, é indicado instalar, juntamente com o banco de capacitores um controlador. Desta forma, o banco de capacitores somente será conectado quando houver necessidade de compensação de energia reativa. $O$ investimento para adequação 
do problema poderá pagar-se em média de seis a oito meses. Para 220V, há capacitores de 10 e 12 KVAR (WEG, [2016]).

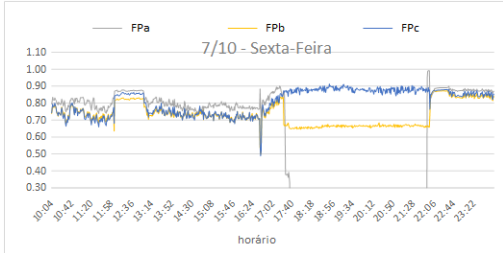

(a)

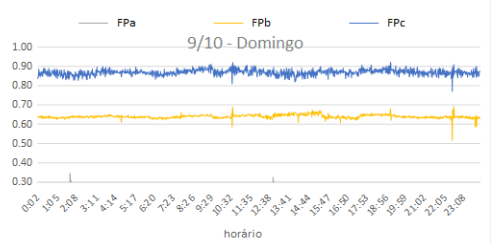

(c)

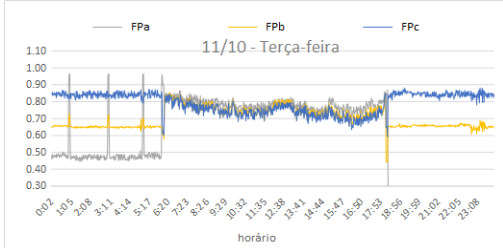

(e)

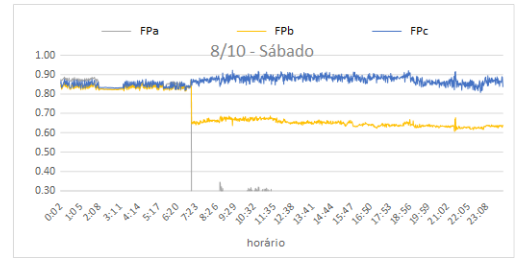

(b)

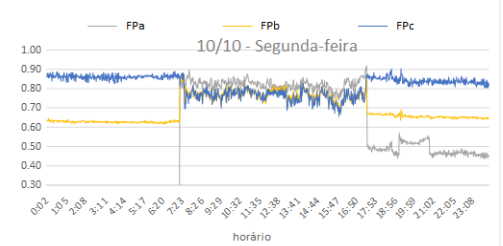

(d)

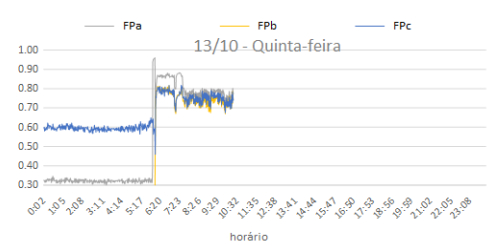

(f)

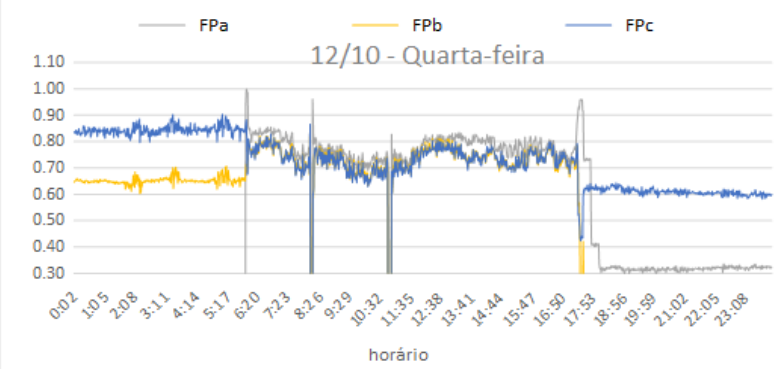

(g)

Figura 10 - $(a)(b)(c)(d)(e)(f)(g)$ Oscilações dos valores de fator de potência FPa,FPb e FPc ao longo do dia. 


\begin{tabular}{|c|c|c|c|c|c|c|c|c|c|c|c|}
\hline & & & & nst & s/Fatc & de & enc & cia & & & \\
\hline & FOP & PONTA & & & & RES & IADO & & To & & \\
\hline & kvarh & $\mathrm{kW}$ & FP & kvan & Wh $F P$ & Wrath & wh & $\mathrm{FP}$ & wath & wh & $F P$ \\
\hline A & 140.81 & 756.480 & 0.96 & -37.474 & $1.267 .0 .06 x$ & 0.000 & 0.000. & 1.00 & 100.33 & 757.77 & 0.29 \\
\hline B & 95.13 & 61304 & 0.00 & .39073 & 1355004 & 0.000 & 0.000 & 100 & 57360 & 61520 & 100 \\
\hline c & 95,154 & 617.56 & 0.00 & -38502 & $1,7 \mathrm{es} \quad 0.0 \mathrm{cc}$ & 0.000 & 0.000 & 1.00 & 50562 & 61935 & 1.00 \\
\hline TOTAL & 331.37 & 199791 & 0.99 & $-114,140$ & 4.407 0.04k & 0.000 & 0.000 & 1.00 & 217.23 & 1902.31 & 0.99 \\
\hline
\end{tabular}

Figura 11 - Relatório dos dados de Fator de Potência com banco de capacitores no software RMS ANAWIN.

Ainda, caso a empresa utilizasse de equipamento com baixo fator de potência, ou seja, obsoletos, poderia ser instalado um banco para a correção do fator de potência junto ao equipamento, o que é chamado de compensação individual.

\section{Considerações Finais}

É importante destacar que apesar da avaliação dos resultados, há inúmeros procedimentos para uma auditoria energética completa, conforme mencionado por diversos autores. No entanto, esse estudo baseou-se apenas na avaliação da qualidade da energia disponibilizada na indústria, de forma que pudesse prover aos autores conhecimentos aplicados de como tratar os dados do analisador e entender determinados parâmetros disponibilizados pelas normas da ANEEL e pelo PRODIST.

Ainda, um estudo completo de auditoria energética deve tomar providências com base nos resultados alcançados, monitorar a melhoria de desempenho após adotadas as medidas propostas e comparar com dados anteriores, o que leva a um estudo mais complexo. Tal estudo é de longa aplicação e, via de regra, exige investimentos de maior porte. Neste caso, devido à crise econômica, 
a empresa não dispõem de caixa para investir em um programa completo de auditoria energética.

Dos resultados obtidos, conclui-se que há algumas medidas de baixo custo que podem assessorar na redução de despesas e aprimoramento da qualidade de energia fornecida no local da planta industrial, a fim de melhorar a vida útil do maquinário, que é o "coração" da indústria metalmecânica gerando maior rentabilidade a longo prazo. Dentre as melhorias podemos destacar:

a) Revisar o TAP do transformador, para a tensão nominal correta.

b) Redistribuir os circuitos da empresa, conforme as cargas das máquinas, reduzindo as sobrecargas na fase $A$.

c) Elaborar um cronograma para ligar o maquinário evitando picos de corrente nos circuitos.

d) Instalar um banco de capacitores de 10 kVAr para correção do fator de potência com um controlador, para melhor gerenciamento dos horários de aplicação do banco.

Por fim, para uma auditoria energética completa, deve-se aplicar essas indicações de otimização da planta industrial, acompanhar o desempenho da planta, e considerar realizar outros diagnósticos como análise da eficiência das máquinas, readequação da iluminação, climatização do local, gerenciamento de ar comprimido, entre outros.

\section{Agradecimentos}

Gostaríamos de deixar nossos agradecimentos à instituição de ensino Fundação Escola Técnica Liberato Salzano Vieira da Cu- 
nha, que nos disponibilizou o analisador de energia MARH-21, equipamento fundamental para a realização deste trabalho. $E$ também à Empresa do estudo de caso, a qual nos proporcionou a oportunidade da coleta de dados.

\section{Referências}

AGÊNCIA NACIONAL DE ENERGIA ELÉTRICA. Resolução no 479, de 03 de abril de 2012: Condições gerais de fornecimento de energia elétrica. Brasília, DF: ANEEL, 2012.

AGÊNCIA NACIONAL DE ENERGIA ELÉTRICA. Resolução no 505, de 26 de novembro de 2001: Níveis de tensão de energia elétrica em regime permanente. Brasília, DF: ANEEL, 2001.

AGÊNCIA NACIONAL DE ENERGIA ELÉTRICA. PRODIST - Módulo 5: Sistemas de Medição. Procedimentos de Distribuição de Energia Elétrica no Sistema Elétrico Nacional. Brasília, DF: ANEEL, 2016. Disponível em: <http://www.aneel.gov.br/MODULO-8>. Acesso em: 13 nov. 2016.

AGÊNCIA NACIONAL DE ENERGIA ELÉTRICA. PRODIST - Módulo 8: Qualidade da Energia Elétrica. Procedimentos de Distribuição de Energia Elétrica no Sistema Elétrico Nacional. Brasília, DF: ANEEL, 2016. Disponível em: <http://www.aneel.gov.br/MODULO8>. Acesso em: 13 nov. 2016.

ALDABÓ, R. Qualidade na energia elétrica. Sāo Paulo: Artliber, 2001. 
BELINOVSKI, K.D.; BISPO, D; SILVA, S.F.P. Análise de eficiência energética em uma indústria de palitos. Uberlândia: Universidade Federal de Uberlândia, 2009.

BRASIL. Ministério do Trabalho e Emprego. Portaria n.598, de 7 de dezembro de de 2004. Cria a norma regulamentadora 10 - Segurança em Instalações e Serviços em Eletricidade. Disponível em: <https://goo.gl/QtrbMj>. Acesso em: 12 dez.2016.

COMITÊ DE DISTRIBUIÇÃO DE ENERGIA ELÉTRICA. Manual de orientação aos consumidores: energia reativa excedente. 2004. Disponível em: <https://goo.gl/t1mX1j.>. Acesso em: 01 jan. 2017.

CREDER, H. Instalações Elétricas. 15.ed. Rio de Janeiro: LTC, 2013.

CRUZ, M.P. Metodologia para avaliação dos impactos econômicos associados a problemas de qualidade de energia. 2007. 164 f. Dissertação (Mestrado em Engenharia Elétrica). Universidade Federal de Santa Catarina - UFSC, Programa de Pós-Graduação em Engenharia Elétrica, Florianópolis, 2007.

ELETROBRÁS. Conservação de energia: Eficiência Energética de Equipamentos e Instalações. 3. ed. Itajubá: Universidade Federal de Itajubá, 2006. Disponível em: <https://goo.gl/cB72TJ>. Acesso em: 12 dez. 2016.

GASPAR, C. Eficiência energética na indústria: Curso de utilização racional de energia. Portugal: Gaia, 2004.

GSI - Engenharia e Consultoria LTDA. MARH-21. São Paulo: GSI, [2017]. 
INTERNATIONAL ELECTROTECHNICAL COMMISSION. IEC 61000/3-4: limites para introdução de corrente harmônicas em sistemas de suprimento de energia elétrica. Genebra: IEC,1998.

INSTITUTE OF ELECTRIC AND ELECTRONIC ENGINEERS. IEEE 519: Requisitos e práticas recomendadas para controle de harmônicas em sistemas de potência elétrica. New York: IEEE, 1992.

INTERNATIONAL ORGANIZATION FOR STANDARDIZATION. ISO 50001: Sistema de Gestão de Energia. Genebra: ISO, 2011.

LIMA, M.A.A. Uma nova metodologia para análise da qualidade da energia elétrica sob condições de ocorrência de múltiplos distúrbios. 2009. 178 f. Tese (Doutorado em Engenharia Elétrica) Universidade de São Paulo, 2009.

LOURENÇO, F.B.M. Optimização Energética numa Unidade Industrial. 2009. 128 f. Dissertação (Mestrado Integrado em Engenharia Elétrica e de Computadores). Faculdade de Engenharia da Universidade do Porto, Porto, 2009.

MAMEDE FILHO, J. Instalações Elétricas Industriais. 8. ed. Rio de Janeiro: LTC, 2015.

MEHL, E. L. M. Qualidade da energia elétrica. Universidade Federal do Paraná, 2012.

NASCIMENTO, D. A. P. Auditoria Energética na Indústria. 2014. 72 f. Monografia (Graduação em Engenharia Elétrica) - Universidade de Brasília, Engenharia em Energia, Brasília, 2014.

OPERADOR NACIONAL DO SISTEMA ELÉTRICO - ONS. Submódulo 2.2: Verificação da conformidade das instalações de transmis- 
são aos requisitos mínimos. Rio de Janeiro: ONS, 2016a. Disponível em: <https://goo.gl/TL4yMq>. Acesso em: 20 nov. 2016.

OPERADOR NACIONAL DO SISTEMA ELÉTRICO- ONS. Submódulo 2.8: Gerenciamento dos indicadores de qualidade da energia elétrica da Rede Básica. Rio de Janeiro: ONS, 2016b. Disponível em: <https://goo.gl//rZ4nxh>. Acesso em: 20 nov. 2016.

PROCEL INDÚSTRIA. Metodologia de realização de diagnóstico energético: guia básico. Brasília: IEL/NC, 2009.

SOPRANO. CATÁlOGo Disj. Caixa Moldada (NEMA). [Caxias do Sul]: SOPRANO, [2011]. Disponível em: <https://goo.gl/sN28KZ>. Acesso em: 12 jan. 2017.

WEG. Automação: Capacitores para Correção do Fator de Potência. Jaguará do Sul: WEG, [2016]. Disponível em: <https://goo.gl/ao7jHH>. Acesso em: 14 jan. 2017. 